\title{
Global Coupled EM-Electrical-Thermal Simulation and Experimental Validation for a Spatial Power Combining MMIC Array
}

\author{
W. Batty ${ }^{\times}$, C. E. Christoffersen ${ }^{+}$, A. B. Yakovlev ${ }^{\circ}$, J. F. Whitaker\#, M. Ozkar*, S. Ortiz*, A. \\ Mortazawi\#, R. Reano\#, K. Yang\#, L. P. B. Katehi\# , C. M. Snowden ${ }^{\times}$and M. B. Steer*, \\ ${ }^{\times}$Institute of Microwaves and Photonics, School of Electronic and Electrical Engineering, \\ University of Leeds, Leeds LS2 9JT, UK. \\ +Department of Electrical Engineering, Lakehead University, 955 Oliver Road, Thunder Bay, \\ Ontario P7B 5E1, Canada. \\ ${ }^{\circ}$ Department of Electrical Engineering, The University of Mississippi, MS 38677-1848, USA. \\ \# Center for Ultrafast Optical Science and Radiation Laboratory, Department of Electrical \\ Engineering and Computer Science, University of Michigan, Ann Arbor, MI, USA. \\ *Department of Electrical and Computer Engineering, North Carolina State University, \\ Raleigh, NC 27695-7914, USA.
}

\begin{abstract}
The first fully coupled electromagneticelectro-thermal global simulation of a large microwave subsystem, here a whole spatial power combining MMIC array, is described. The modeling effort is supported by parallel developments in electro-optic and thermal measurement. The CAD tools and experimental characterisation described, provide a unique capability for the design of quasi-optical systems and for the exploration of the fundamental physics of spatial power combining devices.
\end{abstract}

\section{INTRODUCTION}

Spatial power combining and quasi-optic arrays are being developed as high power sources at millimeter wavelengths. Major advances in device capabilities have been achieved over recent years, with reports of ever higher power outputs and efficiencies, at ever increasing operating frequencies. These advances have been based largely on intuitive and empirical construction, and not the result of intensive computer aided design (CAD). In fact, the complex nature and computational magnitude of the global simulation problem, required to describe spatial power combining systems in their entirety, means that there has been a lack of CAD tools capable of achieving this task.

This paper describes the first application of such a tool to the global coupled electromagnetic (EM)electrical-thermal simulation of a spatial power combining array, and represents the highest level of global modeling reported to date for such a structure. This is combined with description of a joint experimental program capable of validating the global model. These results represent the culmination of several years collaboration on a multi-university research initiative.

Global modelling developments implemented in the simulations described here include: (i) a universal er-

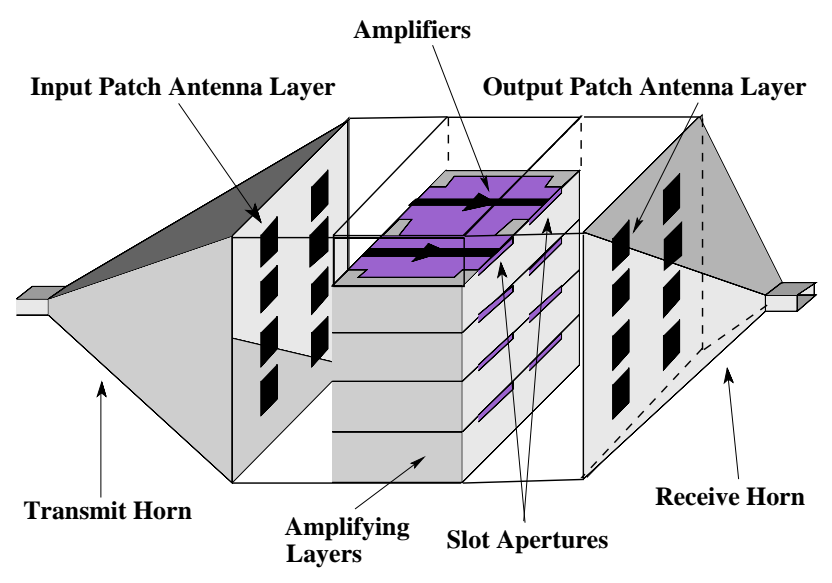

Fig. 1. X-band 'tray/card' MMIC array spatial power combiner used for global EM-electro-thermal simulation and experimental validation.

ror concept based on state variables and local reference node formulations, which facilitates the tight integration of disparate analyses; (ii) a thermal multiport model based on generalized network parameters for non linear thermal systems, giving a fully analytical and minimal thermal impedance matrix description of arbitrarily complex systems, without the use of numerical methods and avoiding the need for all model reduction techniques; (iii) a generalized scattering matrix formulation which enables the cascading of EM partial models, so as to integrate differing EM analyses and develop an EM model of the distributed parts of the array, as well as supporting circuit ports with total current and voltage as required for interfacing to the circuit model; (iv) application of the global environ- 
ment to a very large microwave problem demonstrating the robustness of the approach. In short, this paper presents and validates a philosophy for global modeling of very large problems which includes minimal approximations and close adherence to system physics, as well as readily achieving global convergence.

\section{Global Circuit Simulation}

The power combiner simulated in this study is an X-band 'tray/card' structure introduced in [1], and illustrated schematically in Fig. 1. The actual structure modeled is a $5 \times 5$ MMIC array in an aluminium tray framework of size $12 \mathrm{~cm} \times 9 \mathrm{~cm} \times 5 \mathrm{~cm}$.

Explicitly coupled global EM-electro-thermal circuit level simulation of this device is achieved by employing microwave circuit simulator, Transim, developed at NCSU [2]. This object-oriented code implements a global simulation approach in which concurrent EM, circuit and thermal analyses are supported. The highlevel circuit abstraction is retained, and the results of EM and thermal analysis for the spatially distributed physical subdomains, are incorporated into the circuit framework as EM Y-parameters and generalised thermal Z-parameters, respectively. This network based global circuit simulation approach is described in [3].

Transim describes non linear devices based on state variables which provides great flexibility for the design of new models. The addition of new circuit element models and analysis types into Transim is therefore much simpler than for other circuit simulators. For example, new element models are coded and incorporated into the program without modification to the high-level simulator. The circuit analysis types currently available in Transim are DC, AC, state variable harmonic balance (HB), convolution transient, wavelet transient, and time-marching transient [4].

Transim employs a local reference node concept [5] which was initially developed for combined circuit and $\mathrm{EM}$ analysis of distributed microwave circuits, and also guarantees that there is no mixing of electric and thermal currents. This fundamental feature is utilized here for the analysis of spatially distributed circuits as well as for the integration of disparate simulation domains.

The use of state variables, with network parameters for distributed systems, and combined with a universal error concept, means that Transim can implement virtually any physical description in its modeling scheme, with the number of non linear unknowns generally much smaller than the number of unknowns in conventional circuit analysis. This results in an important reduction in computation time.

\section{Thermal Impedance Matrix Model}

The fully analytical thermal impedance matrix model, developed at Leeds University for the treatment of the global electro-thermal modeling problem, is now described briefly [6], [7]. The Leeds model uses the Kirchhoff transformation and a subsequent time variable transformation to linearize the non linear time-dependent heat diffusion equation, and solves the transformed equation fully analytically in complex volumes, by domain decomposition [7]. The thermal impedance matrix approach then reduces to the construction of global heat flow functions, $R_{T H_{i j}}(s)$, for power dissipating and temperature sensitive elements in semiconductor integrated circuits, relating Laplace transformed temperature rise of element $i$ above its initial temperature, to Laplace transformed fluxes due to power dissipation in elements, $j$.

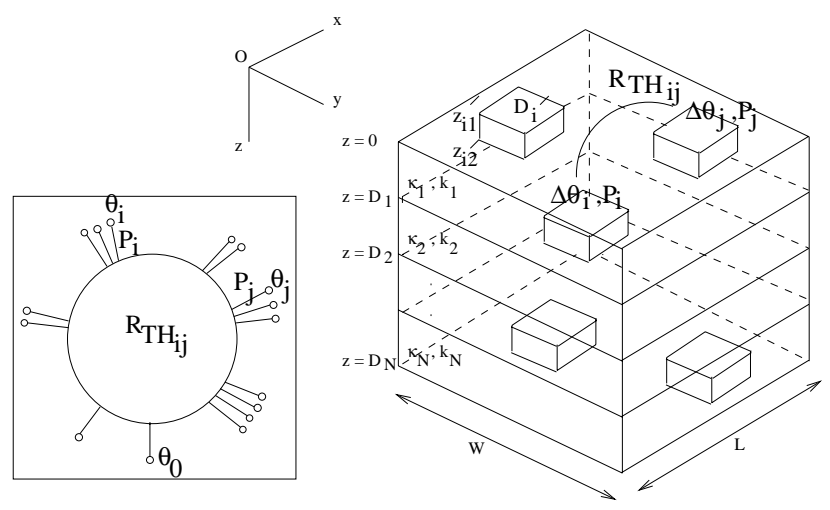

Fig. 2. N-level multilayer with arbitrarily distributed volume sources for fully analytical construction of thermal impedance matrix $R_{T H_{i j}}(s)$. Inset: N-port described by generalised multiport thermal Z-parameters, $R_{T H_{i j}}(s)$.

The most general analytical subvolume solution is constructed for the generic thermal multilayer of Fig. 2 . For illustration, the corresponding form of the thermal impedance matrix for a single layer is,

$$
\begin{array}{r}
R_{T H_{i j}}(s)=\frac{1}{\kappa_{S} L W} \sum_{m n} \frac{I_{m n}^{i} I_{m n}^{j}}{I_{00}^{i}} \frac{-4}{\left(1+\delta_{m 0}\right)\left(1+\delta_{n 0}\right)} \frac{1}{\gamma_{m n}^{2}} \\
\times\left[\begin{array}{c}
\left.\frac{\sinh \gamma_{m n} z_{i 2}-\sinh \gamma_{m n} z_{i 1}}{\gamma_{m n}\left(z_{i 2}-z_{i 1}\right)} \times \cosh \gamma_{m n}\left(D-z_{i 2}\right)\right] \\
\frac{\left[\cosh \gamma_{m n}\left(D-z_{i 1}\right)-\cosh \gamma_{m n}\right.}{\cosh \gamma_{m n} D} \\
+1-\frac{\sinh \gamma_{m n}\left(z_{i 2}-z_{i 1}\right)}{\gamma_{m n}\left(z_{i 2}-z_{i 1}\right)}
\end{array}\right] .
\end{array}
$$

Here, $z_{i 1}, z_{i 2}$ are the $z$-coordinates of the planes bounding heat dissipating volume, $i$, in the $z$-direction. $D_{i}$ is the corresponding $x-y$ cross-section, and

$$
\begin{array}{r}
\lambda_{m}=\frac{m \pi}{L}, \quad \mu_{n}=\frac{n \pi}{W}, \quad \gamma_{m n}^{2}=\lambda_{m}^{2}+\mu_{n}^{2}+\frac{s}{k_{S}}, \\
I_{m n}^{i}=\iint_{D_{i}} \cos \left(\frac{m \pi x}{L}\right) \cos \left(\frac{n \pi y}{W}\right) d x d y,
\end{array}
$$


with $m, n=0,1,2, \ldots . R_{T H_{i j}}(s)$ then represents the generalised multiport thermal Z-parameters for a thermal N-port, Fig. 2 (inset), with the Z-parameters evaluated either directly in frequency space, $s \rightarrow j \omega$, giving the impulse response, $R_{T H_{i j}}(j \omega)$, or by analytical or numerical Laplace inversion in the time-domain, giving the step response, $R_{T H_{i j}}(\tau)=\mathcal{L}^{-1}\left\{R_{T H_{i j}}(s) \frac{1}{s}\right\}$ [7]. By netlist combination of such thermal subvolume solutions, this approach readily includes MMIC structural detail such as surface metallisation, vias and airbridges, as well as global structure at the level of the whole spatial power combiner array.

\section{EM Admittance Matrix Model}

The EM precomputation, required for description of a structure such as the X-band array considered here, is now outlined.

The EM modeling environment for the full-wave analysis of a waveguide-based aperture-coupled patch amplifier array, Fig. 1, is based on a decomposition of the entire structure into electromagnetically-coupled modules. This includes transmit and receive rectangular waveguide tapers attached to the N-port aperturecoupled patch array waveguide transitions (divider and combiner), waveguide-to-microstrip line junctions, and amplifier circuits. Each module is simulated using the most efficient numerical technique resulting in the generalized scattering matrix (GSM) of propagating and evanescent modes. An overall response of the entire array is obtained by cascading the responses of the individual modules.

The EM algorithm for complete modeling of the amplifier array was described in detail in [8]. In particular, an integral equation formulation for the induced electric and magnetic currents discretized via the method of moments was developed for a waveguideto-aperture-coupled patch array, resulting in the GSM of the N-port spatial power divider/combiner. The GSM for a rectangular waveguide taper approximated by double-plane stepped junctions was obtained using a mode-matching technique. A three-dimensional commercial finite element method program was used to model waveguide-to-microstrip line transitions. It should be noted that the GSM approach utilized here enables the cascading of EM partial modules as well as supporting circuit ports with total current and voltage as required for interfacing to the circuit model.

Thermal and EM precomputation of network parameters then allow fully coupled EM-electro-thermal global simulation on CAD timescales.

\section{EXPERIMENTAL VALIDATION}

The simulation program discussed above has been conducted jointly, and developed iteratively, with a program of experimental characterisation, both EM and thermal. During the past several years, a researchprototype electro-optic (EO) near-field imaging system has been under development at the University of Michigan. The EO field-mapping technique, in which various electro-optic crystals have been utilized as both electric field and temperature sensors, has been demonstrated to provide a thorough picture of the physical phenomena underlying the operation of a variety of microwave integrated circuits, antennas, and arrays [9], [10].

Spatial resolution has been demonstrated to be on the order of $5 \mu \mathrm{m}$, and the extremely fast response of an EO crystal to a time-varying field, along with the use of ultrashort-pulse-duration lasers to probe the effect of the electric-field on the EO crystal, has allowed the demonstration of a measurement bandwidth for the field-mapping system that exceeds 100 $\mathrm{GHz}$. In addition, it has proved possible to map three vectorial field components separately, and to obtain the amplitude and phase of the electric field simultaneously during the imaging process. Finally, a non-contact measurement configuration combined with optical-fiber-coupled, low-dielectric-constant EO crystals [11] makes the field-mapping system less intrusive to a device under test than any other near-field measurement method. Field maps generated using such techniques can be compared against EM simulations.

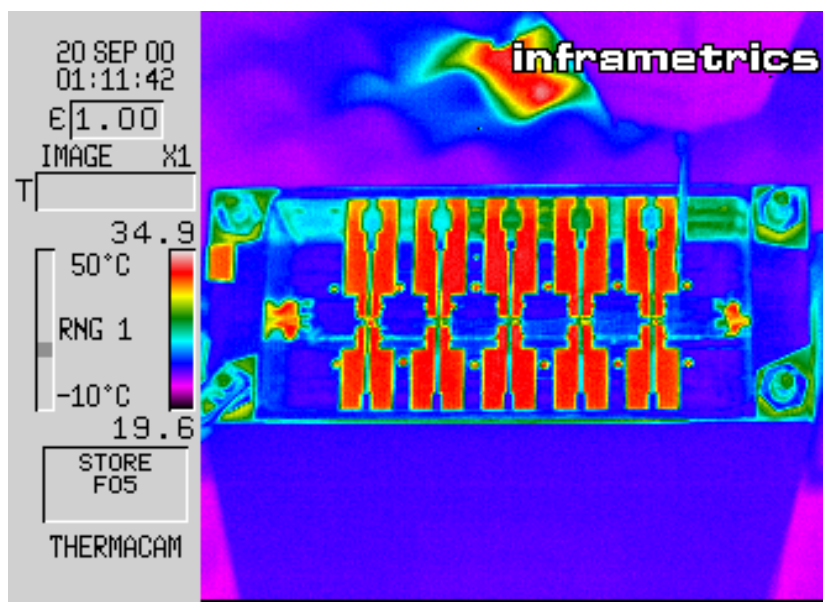

Fig. 3. Infrared thermal image of a lateral cross-section of the $\mathrm{X}$-band 'tray/card' MMIC array, showing 5 MMIC die.

It is also possible for the EO system to simultaneously map both electric field and temperature distributions, by utilising an optical absorption modulation that takes place at certain probe-laser wave- 
lengths within semiconductor EO crystals. This allows for the combined electro-thermal examination of active microwave and millimeter-wave circuits with a single probe and the ability to calibrate electric field data that is corrupted when the probe is placed in areas where temperature variations are present. The technique has been used to compare electric and thermal fields on a patch antenna radiating high-power [12], and it is now being applied to the combined electro-thermal examination of the microwave elements in the quasi-optical power-combining array described here.

Infrared thermal images of the X-band spatial power combiner have also been obtained using an Inframetrics ThermaCam, Fig. 3. Allowing for the difficulties of emissivity correction and specification of external heat flux coefficients, and uncertainties in device and material parameters, agreement between experiment and simulations is found to be reasonable, partially validating the global model.

\section{Results}

Fig. 4 shows the results of a coupled EM-electrothermal, single-tone HB simulation of the spatial power combining array. The figure illustrates the impact of thermal effects on total system output voltage waveform at the output waveguide. The calculated response is sensitive to the thermal boundary conditions employed in describing the MMIC array. These are de-

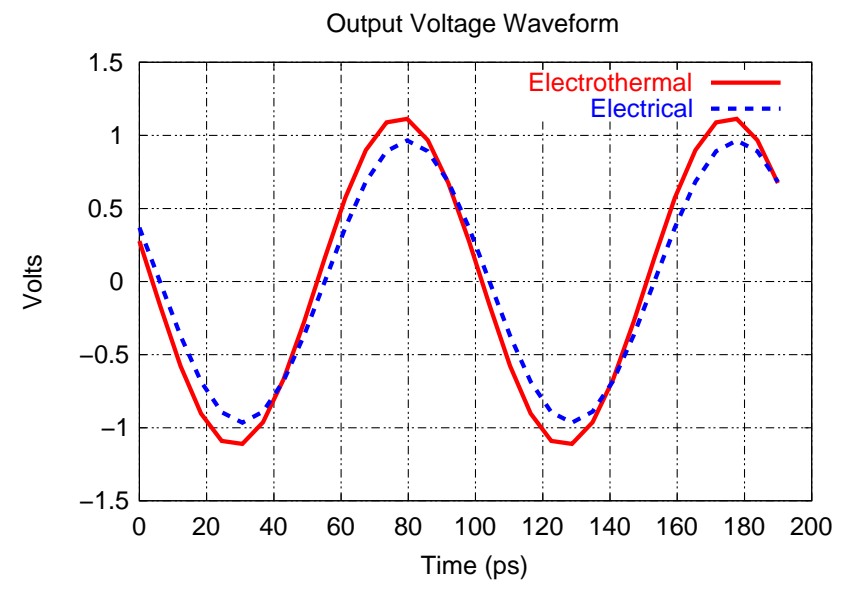

Fig. 4. Coupled EM-electro-thermal, single-tone HB simulation of the spatial power combining MMIC array, illustrating thermal effects on total system output.

pendent on surface emissivity which is not a well specified parameter. Previous simulations have shown that transient rise time to steady-state, and the steady-state temperature achieved, are both highly sensitive to the value of surface emissivity and to the resulting ratio of radiative to convective losses.

\section{Conclusion}

The first global simulation of a whole spatial power combining array has been described and spot validated against experimental measurement. The CAD tool and experimental characterisation capability developed will be used in the design and study of spatial power combiners for use as high power millimeter-wave sources.

\section{ACKNOWLEDGEMENT}

This work was supported by the U.S. Army Research Office through Clemson University as a Multidisciplinary Research Initiative on Quasi-Optics, agreement Number DAAG55-97-K-0132.

\section{REFERENCES}

[1] S. Ortiz and A. Mortazawi, "A perpendicularly-fed patch array for quasi-optical power combining," 1999 MTT-S IEEE Int. Microwave Symp. Dig., pp. 667-670, June 1999.

[2] C. E. Christoffersen, U. A. Mughal, M. B. Steer, "Object oriented microwave circuit simulation," Int. J. RF and $M i$ crowave CAE, Vol. 10, No. 3, pp. 164-182, 2000.

[3] M. B. Steer, J. F. Harvey, J. W. Mink, M. N. Abdulla, C. E. Christoffersen, H. M. Gutierrez, P. L. Heron, C. W. Hicks, A. I. Khalil, U. A. Mughal, S. Nakazawa, T. W. Nuteson, J. Patwardhan, S. G. Skaggs, M. A. Summers, S. Wang and A. B. Yakovlev, "Global modeling of spatially distributed microwave and millimeter-wave systems," IEEE Trans. Microwave Theory Tech., Vol. 47, pp. 830-839, 1999.

[4] C. E. Christoffersen, Global modeling of nonlinear microwave circuits, Ph.D. Dissertation, North Carolina State University, Dec. 2000.

[5] C. E. Christoffersen and M. B. Steer, "Implementation of the local reference concept for spatially distributed circuits," Int. J. of RF and Microwave Computer-Aided Eng., Vol. 9, No. 5, 1999.

[6] W. Batty, A.J. Panks, R.G. Johnson and C.M. Snowden, "Electro-thermal modeling of monolithic and hybrid microwave and millimeter wave IC's," VLSI Design, Vol. 10, No. 4, pp. 355-389, 2000.

[7] W. Batty, C. E. Christoffersen, A. J. Panks, S. David, C. M. Snowden and M. B. Steer, "Electro-thermal CAD of power devices and circuits with fully physical timedependent compact thermal modeling of complex non linear 3-d systems," IEEE Trans. Comp. Packag. Technol., to be published, Dec. 2001.

[8] A. B. Yakovlev, S. Ortiz, M. Ozkar, A. Mortazawi, and M. B. Steer, "A waveguide-based aperture-coupled patch amplifier array - full-wave system analysis and experimental validation," IEEE Trans. Microwave Theory Tech., Vol. 48, pp. 2692-2699, Dec. 2000.

[9] K. Yang, G. David, S. Robertson, J. F. Whitaker, and L. P. B. Katehi, "Electro-optic mapping of near-field distributions in integrated microwave circuits," IEEE Trans. Microwave Theory Tech., Vol. 46, pp. 2338-2343, Dec. 1998.

[10] K. Yang, T. Marshall, M. Forman, J. Hubert, L. Mirth, Z. Popovic, L. P. B. Katehi, and J. F. Whitaker, "Activeamplifier-array diagnostics using high-resolution electrooptic field mapping," IEEE Trans. Microwave Theory Tech., Vol. 49, pp. 849-857, May 2001.

[11] K. Yang, L. P. B. Katehi, and J. F. Whitaker, "Electricfield mapping system using an optical-fiber-based electrooptic probe," IEEE Microwave Wireless Comp. Lett., Vol. 11, pp. 164-166, Apr. 2001.

[12] R. M. Reano, K. Yang, J. F. Whitaker, and L. P. B. Katehi, "Integrated electro-thermal probe," accepted for publication in IEEE Trans. Microwave Theory Tech. 